\title{
A note on measurement of sound pressure with intensity probes
}

\author{
Juhl, Peter; Jacobsen, Finn
}

Published in:

Journal of the Acoustical Society of America

Link to article, DOI:

$10.1121 / 1.1777851$

Publication date:

2004

Document Version

Publisher's PDF, also known as Version of record

Link back to DTU Orbit

Citation (APA):

Juhl, P., \& Jacobsen, F. (2004). A note on measurement of sound pressure with intensity probes. Journal of the Acoustical Society of America, 116, 1614-1620. https://doi.org/10.1121/1.1777851

\section{General rights}

Copyright and moral rights for the publications made accessible in the public portal are retained by the authors and/or other copyright owners and it is a condition of accessing publications that users recognise and abide by the legal requirements associated with these rights.

- Users may download and print one copy of any publication from the public portal for the purpose of private study or research.

- You may not further distribute the material or use it for any profit-making activity or commercial gain

- You may freely distribute the URL identifying the publication in the public portal

If you believe that this document breaches copyright please contact us providing details, and we will remove access to the work immediately and investigate your claim. 


\title{
A note on measurement of sound pressure with intensity probes $^{\text {a) }}$
}

\author{
Peter Juhl ${ }^{\text {b) }}$ \\ Physics Department, University of Southern Denmark, Campusvej 55, DK-5230 Odense M, Denmark \\ Finn Jacobsen ${ }^{\mathrm{c})}$ \\ Acoustic Technology, Ørsted DTU, Technical University of Denmark, Building 352, Ørsteds Plads, \\ DK-2800 Kgs. Lyngby, Denmark
}

(Received 1 December 2003; revised 8 March 2004; accepted 8 June 2004)

\begin{abstract}
The effect of scattering and diffraction on measurement of sound pressure with "two-microphone" sound intensity probes is examined using an axisymmetric boundary element model of the probe. Whereas it has been shown a few years ago that the sound intensity estimated with a two-microphone probe is reliable up to $10 \mathrm{kHz}$ when using $0.5 \mathrm{in}$. microphones in the usual face-to-face arrangement separated by a $12 \mathrm{~mm}$ spacer, the sound pressure measured with the same instrument will typically be underestimated at high frequencies. It is shown in this paper that the estimate of the sound pressure can be improved under a variety of realistic sound field conditions by applying a different weighting of the two pressure signals from the probe. The improved intensity probe can measure the sound pressure more accurately at high frequencies than an ordinary sound intensity probe or an ordinary sound level meter. (c) 2004 Acoustical Society of America. [DOI: $10.1121 / 1.1777851]$
\end{abstract}

PACS numbers: 43.58.Fm, 43.50.Yw, 43.38.Kb [AJZ] Pages: 1614-1620

\section{INTRODUCTION}

The dominating principle for measurement of sound intensity in air employs two closely spaced pressure microphones and is based on approximating the gradient of the sound pressure by a finite difference. ${ }^{1}$ In the early 1980s it was shown experimentally that the face-to-face configuration with a solid spacer between the two microphones is particularly favorable. ${ }^{2}$ In 1998 it was found that the effect of scattering and diffraction tends to counterbalance the finitedifference error under virtually any sound field condition that can be encountered in practice. ${ }^{3}$ It was shown that the practical upper frequency limit of a sound intensity probe based on two $0.5 \mathrm{in}$. microphones separated by a $12 \mathrm{~mm}$ spacer in the face-to-face arrangement is about $10 \mathrm{kHz},{ }^{3}$ which is about an octave higher than the frequency limit determined by the finite-difference approximation. ${ }^{1,2}$

One of the most important indicators for evaluating the reliability of a sound intensity or sound power measurement is the pressure-intensity index (the normalized ratio of the mean square pressure to the intensity in logarithmic form). This quantity, which can be determined with the intensity probe during the measurement, ${ }^{1}$ reflects the acoustic conditions of the measurement and provides important information about the influence of phase mismatch. ${ }^{1,4}$ Determining the pressure-intensity index obviously involves measuring the sound pressure. However, experimental results have indicated that the sound pressure level measured with a typical intensity probe tends to be too low at high frequencies even

\footnotetext{
${ }^{a)}$ Portions of this work were presented in "Sound pressure measurements with sound intensity probes," Proceedings of 18th International Congress on Acoustics, Kyoto, Japan, April 2004.

${ }^{b}$ Electronic mail: pmjuhl@fysik.sdu.dk

${ }^{c}$ Electronic mail: fja@oersted.dtu.dk
}

though the sound intensity is accurately estimated. ${ }^{5}$ Underestimation of the pressure-intensity index may cause the operator to accept inaccurate measurements. Thus, the purpose of this paper is to examine how the sound pressure is estimated by a conventional intensity probe in various sound fields, and, if possible, to improve the estimate.

\section{THE FINITE-DIFFERENCE ERROR}

The complex sound pressure at the center of the probe is estimated as

$$
\hat{p}=\left(\hat{p}_{1}+\hat{p}_{2}\right) / 2,
$$

where $\hat{p}_{1}$ and $\hat{p}_{2}$ are the pressure signals as sensed by the microphones of the probe. Here and in what follows the caret indicates an estimated quantity, which may differ from "true" value because of diffraction, scattering, and the finite difference approximation. The axial component of the complex particle velocity (the component in the direction of the line that joins the acoustic centers of the two microphones) is

$$
\hat{v}_{r}=\left(\hat{p}_{1}-\hat{p}_{2}\right) /(j \rho c k \Delta r),
$$

where $c$ is the speed of sound, $\rho$ is the density of air, $k$ is the wave number, and $\Delta r$ is the acoustic distance between the microphones. This leads to the following expression for the estimated sound intensity: ${ }^{1}$

$$
\hat{I}_{r}=\operatorname{Im}\left\{\hat{p}_{1} \hat{p}_{2}^{*}\right\} /(2 \rho c k \Delta r) .
$$

The finite-difference error of the intensity is due to the combined effect of Eqs. (1) and (2), i.e., the effect of replacing the pressure at the center of the probe with the arithmetic mean of the two pressure signals and replacing the true pressure gradient $\partial p / \partial r$ with the finite difference $\Delta p / \Delta r$ (although the term "finite-difference error" seems to refer only 


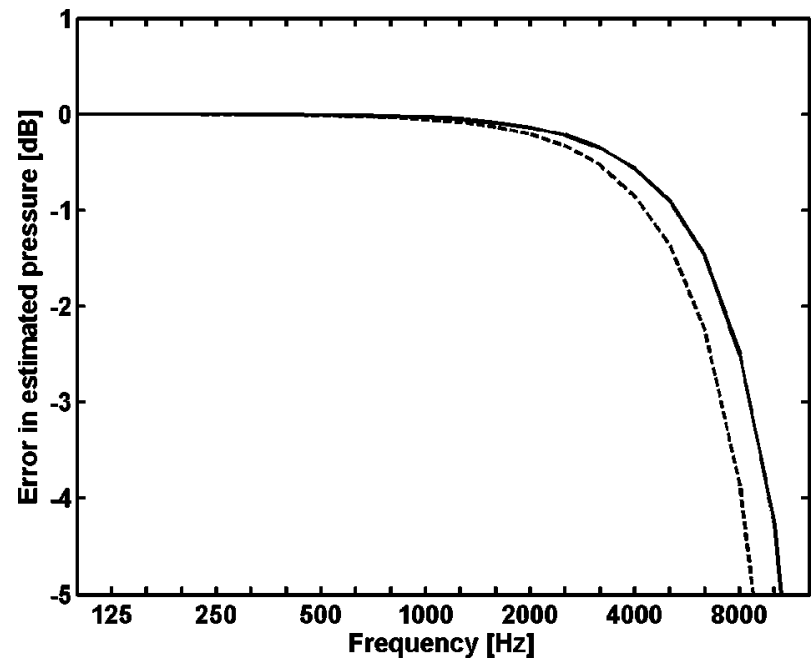

FIG. 1. Finite-difference approximation error in a plane wave of axial incidence with a microphone separation distance of $12 \mathrm{~mm} .-$, sound intensity; ---, sound pressure.

to the latter approximation). For a plane wave of axial incidence the finite-difference error in the estimated intensity (the ratio of the estimate to the true intensity ignoring scattering and diffraction) can be shown to be ${ }^{2}$

$$
\hat{I}_{r} / I_{r}=\frac{\sin (k \Delta r)}{k \Delta r},
$$

whereas the finite-difference error in the estimated pressure is $^{6}$

$$
\hat{p} / p=\cos (k \Delta r / 2) .
$$

As can be seen from Fig. 1, both expressions indicate underestimation at high frequencies. However, the numerical and experimental study described in Ref. 3 demonstrated that for a wide range of sound field conditions scattering and diffraction effects almost completely cancel the finite-difference error of the intensity.

With a $12 \mathrm{~mm}$ spacer between the microphones Eq. (5) predicts a finite-difference error of the sound pressure of about $1 \mathrm{~dB}$ at $4.3 \mathrm{kHz}$ in a plane wave of axial incidence. However, it may be expected that the increase of the pressure due to scattering and diffraction will compensate for the finite-difference error at least to some extent.

\section{A BOUNDARY ELEMENT MODEL}

The boundary element method (BEM) based on the Helmholtz integral equation is particularly suited for solving acoustic scattering and diffraction problems. The present work evolves from earlier work. In Ref. 7 an axisymmetric BEM model of a condenser microphone was presented, and it was shown how the outcome of a BEM calculation, which both then and in the present case is a pressure distribution over the "diaphragm" of the "microphone," could be related to the electrical output of the device relative to its pressurefield response (i.e., the free-field correction was calculated). At frequencies more than an octave below the resonance of the microphone, which is the frequency range considered here, it was found that the difference between the pressure-

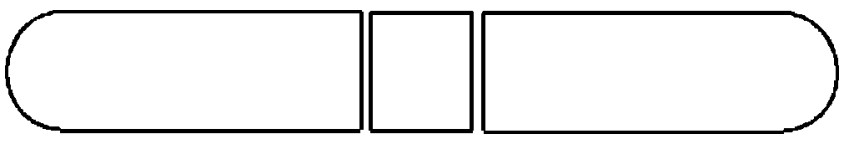

FIG. 2. Geometry of sound intensity probe used in the numerical model.

field response and the free-field response was almost exclusively due to diffraction. The effect due to the finite impedance of the diaphragm was found to be less than $0.1 \mathrm{~dB}$ in this frequency range, and this effect is therefore ignored in the present work. Although the model of the microphone is axisymmetric, nonaxisymmetric sound fields (such as plane wave incidence from other directions than the microphones' axis of rotation) can be handled by taking advantage of the fact that a nonaxisymmetric diaphragm movement does not result in an electrical signal from the microphone. ${ }^{7}$ The accuracy of the BEM calculation for a single microphone was established by comparing the calculations with very accurate experimental results. ${ }^{7}$

The BEM model was extended to calculations for an intensity probe in Refs. 3, 8, and 9, and this model has been used in the present work. The probe is modeled as a cylinder with a diameter of $12.7 \mathrm{~mm}$, rounded at both ends. Each "microphone" is $31.8 \mathrm{~mm}$ long; there is a 1.1-mm gap between the "diaphragm" and the spacer; and the "12 mm spacer" is actually $10.9 \mathrm{~mm}$ long-see Fig. 2. The two microphones and the spacer were modeled using 69 quadratic elements and 141 nodes. At $12.5 \mathrm{kHz}$, which is the upper frequency limit of the present study, this corresponds to about 22 nodes per wavelength, which is safely above the rule of thumb of using six nodes per wavelength. All frequencies considered in the following are below the first characteristic frequency of the boundary element formulation, ${ }^{10,11}$ and therefore no special care has been taken to avoid the nonuniqueness problem.

The model of an intensity probe may give rise to numerical problems because of the close surfaces of the microphones and the spacer. This problem was addressed in Ref. 8, where a method of dealing with such close surfaces was presented and validated. The use of the model for simulation of intensity probe in sound fields of practical relevance was presented in Ref. 3, in which the predictions of the numerical model were found to be in very good agreement with experimental results.

Figure 3, which is adapted from Ref. 12, presents a comparison between measured values of the weighted pressure increase at the two microphones in the probe (the two freefield corrections) and the corresponding calculations for a plane wave at axial incidence. It can be seen that there is excellent agreement between the measurements and the calculations - the maximum deviation is about $0.1 \mathrm{~dB}$.

One of the purposes of the spacer is to ensure a welldefined acoustic distance between the acoustic centers of the two microphones. For a plane wave at axial incidence the phase difference between the two pressure signals should ideally be

$$
\Delta \varphi=k \Delta r,
$$

where $\Delta r$, the (acoustic) separation distance, obviously 


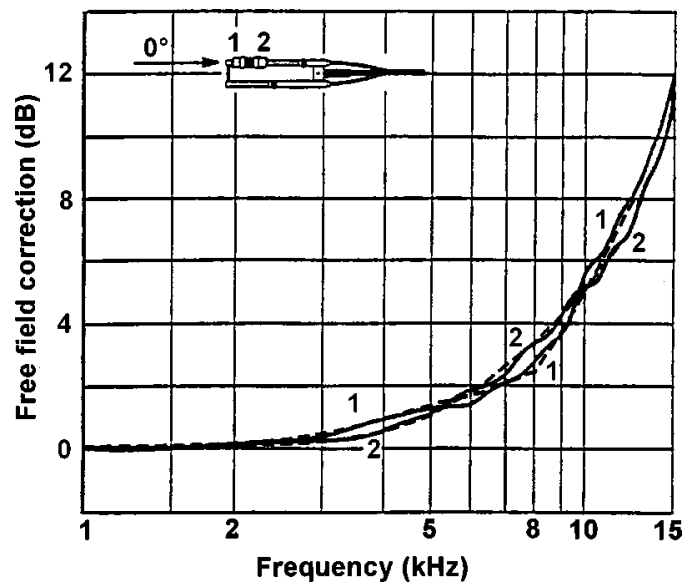

FIG. 3. Free-field correction of the two microphones of a sound intensity probe with $0.5 \mathrm{in}$. microphones separated by a $12 \mathrm{~mm}$ spacer for axial plane wave incidence.-, experimental results reproduced from Ref. 12; ---, numerical results.

should be independent of the frequency. In Fig. 4, which is adapted from Ref. 12, the effective separation distance predicted by the calculations is compared with measured values. The figure shows twice the value of $\Delta r$, and the agreement between measurements and calculations is very good: the maximum deviation occurs at $6 \mathrm{kHz}$ and is about $0.5 \mathrm{~mm}$, which corresponds to a relative deviation of about $2 \%$ between the predicted and measured phase difference.

\section{NUMERICAL RESULTS}

The results presented in what follows have been calculated for an intensity probe with two $0.5 \mathrm{in}$. microphones separated by a $12 \mathrm{~mm}$ spacer (see Fig. 2). The calculations have been carried out at the one-third octave center frequencies from $100 \mathrm{~Hz}$ to $12.5 \mathrm{kHz}$.

\section{A. The conventional sound pressure estimate}

Figure 5 shows the combined effect of the finitedifference error and diffraction on the estimated sound pressure for a plane wave at various angles of incidence. The model of the probe is perfectly symmetrical about a plane though the middle of the spacer with a normal along the axis of rotation. Because of this symmetry the response at a certain angle of incidence, $\theta$ (where $\theta=0$ indicates axial incidence), is identical with the response at the supplementary

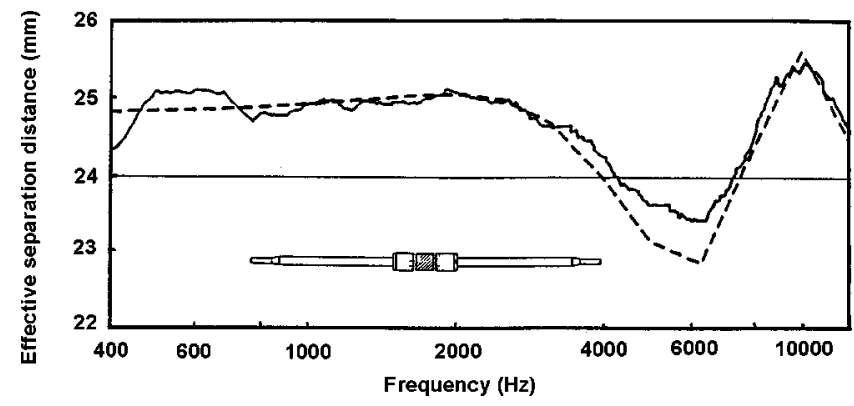

FIG. 4. Effective separation distance (multiplied by 2) between the two microphones of a sound intensity probe with $0.5 \mathrm{in}$. microphones separated by a $12 \mathrm{~mm}$ spacer for axial plane wave incidence.-, experimental results reproduced from Ref. 12; ---, numerical results.

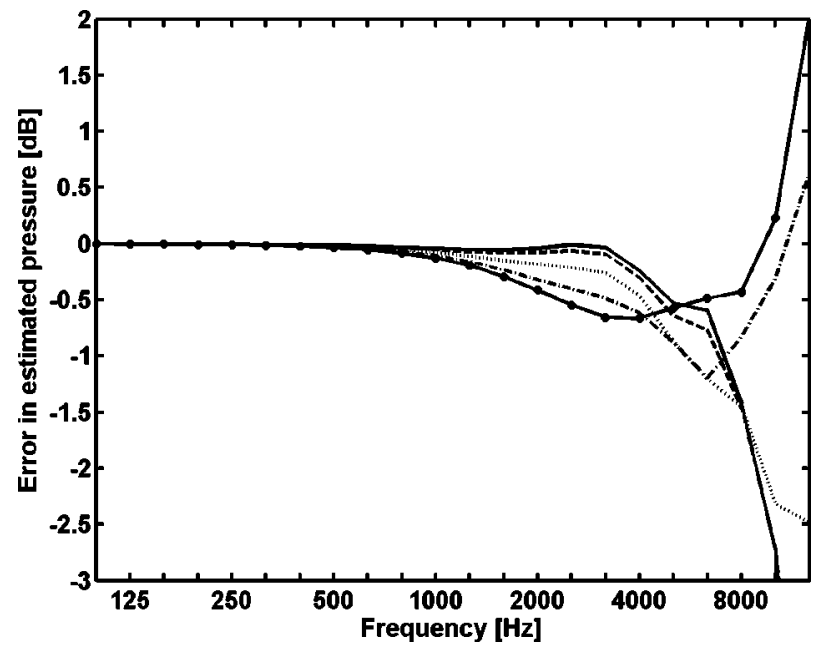

FIG. 5. Error in the estimate of the sound pressure for a plane wave at various angles of incidence:- $0^{\circ} ;---, 20^{\circ} ; \cdots, 40^{\circ} ;-\cdots-, 60^{\circ} ;-1,80^{\circ}$.

angle of incidence, $\pi-\theta$; therefore angles of incidence only up to $80^{\circ}$ are shown. It is clear from Fig. 5 that the pressure increase due to diffraction partly compensates for the finitedifference error-for axial incidence the $1 \mathrm{~dB}$ limit is reached just below $8 \mathrm{kHz}$ (compare with Fig. 1). It is also apparent that at $10 \mathrm{kHz}$, which is the upper limiting frequency for sound intensity measurements with a probe with 0.5 in. microphones separated by a $12 \mathrm{~mm} \mathrm{spacer,}{ }^{3}$ the sound pressure of a plane wave of axial incidence will be underestimated by about $3 \mathrm{~dB}$.

\section{B. An improved estimate}

Although the estimate of the pressure given by Eq. (1) works very well in estimating the intensity as shown in Ref. 3 , it might be possible to develop a better estimate for the pressure itself. A simple estimate could be based on a weighted average, as follows:

$$
\hat{p}_{w}=\left(w \hat{p}_{1}+(1-w) \hat{p}_{2}\right), \quad w \in[0 ; 1] .
$$

The sum of the two weighting factors must obviously equal unity for the estimate to be valid at low frequencies. In order to find an optimum value for $w$ a function to be minimized must be established. Such a function could be

$$
G(w)=\int_{0}^{\pi / 2}\left|\hat{p}_{w}\right| f_{w}(\theta) d \theta /\left(|p| \int_{0}^{\pi / 2} f_{w}(\theta) d \theta\right),
$$

which ideally should equal one ( $p$ is the sound pressure in the undisturbed field). Because of the symmetry of the probe and the fact that two pressure responses from the individual microphones are almost identical, the estimated sound pressure for a plane wave incident at a certain angle, $\theta$, is almost identical with the estimated sound pressure at $\pi-\theta$, in spite of the different weighting of the two signals. The weighting factor $f_{w}(\theta)$ gives the possibility of favoring estimates that work particularly well in a certain direction, for example, near axial incidence, and this may be advantageous since the probe is pointing towards the sound source in typical sound power measurements. Figure 6 shows the maximum absolute value of $20 \log (G(w))$ in the frequency range from $2 \mathrm{kHz}$ to 


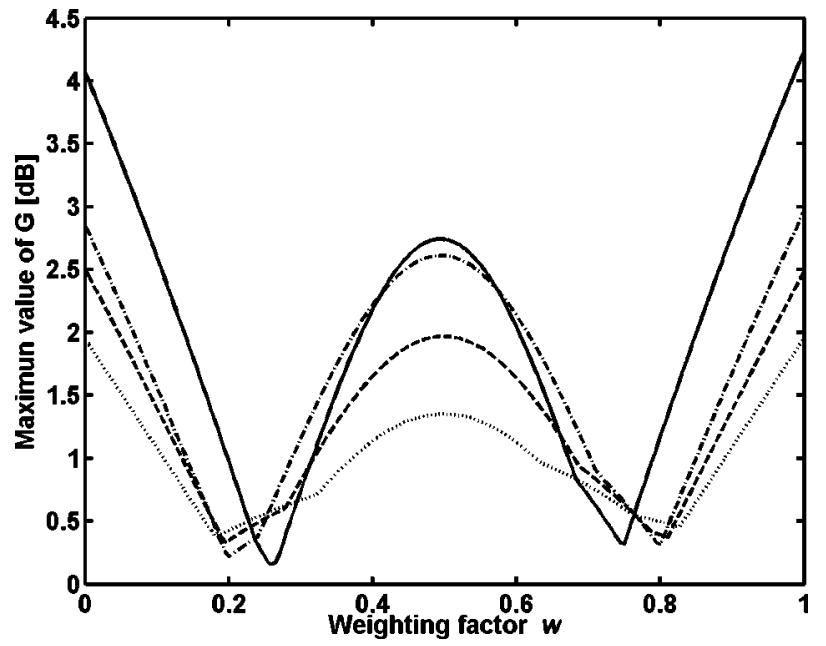

FIG. 6. Maximum value of the logarithmic magnitude of the cost function $G$ in the frequency range $2-10 \mathrm{kHz}$ as a function of the weighting factor $w$. Weighting function:- $\delta(\theta) ;---, \cos (\theta) ; \cdots, 1 ;-\cdots, 1-H(\theta-\pi / 4)$.

$10 \mathrm{kHz}$ as a function of the weighting parameter $w$ calculated for several different weighting functions. At frequencies below $2 \mathrm{kHz}$ all estimates give good results.

Four different weighting functions are examined in Fig. 6. Because the two sound pressure signals are very similar all the curves are almost symmetrical around $w=\frac{1}{2}$. It can be seen that the conventional estimate of the sound pressure corresponding to $w=\frac{1}{2}$ is not the optimal estimate with any of the weighting functions. The solid curve has been calculated with the weighting $\delta(\theta)$, which corresponds to optimizing the response for axial incidence. The dashed line corresponds to a weighting of $\cos (\theta)$, which favors near-axial incidence in the optimization; the dotted curve corresponds to an equal weighting of all angles of incidence between 0 and $\pi / 2$; and finally the dashed-dotted curve corresponds to an equal weighting of all angles between 0 and $\pi / 4$ in the optimization ( $H$ is the Heavyside function). It is apparent that the optimum value of $w$ depends on the sound field, as one would expect. However, it can also be seen that values of $w$ around 0.2 or 0.8 improve the estimate of the sound pressure significantly for all weighting functions compared with the conventional estimate with $w=\frac{1}{2}$.

Figure 7 shows the error of the pressure estimate with $w=0.775$, which seems to be close to the optimum value for all the weighting functions considered here. The figure demonstrates that the estimate with $w=0.775$ performs significantly better than the conventional one (compare with Fig. 5) in the sound fields examined here. In the frequency range of concern (up to $10 \mathrm{kHz}$ ) the error is less than $1.1 \mathrm{~dB}$, and for near-axial incidence the error is less that $0.2 \mathrm{~dB}$ in the frequency range up to $8 \mathrm{kHz}$. Moreover, both positive and negative errors occur, and this will probably cause the errors to cancel out at least to some extent in a typical measurement situation with a sound intensity probe.

It is interesting to compare the performance of the improved estimate with that of a single microphone of the same dimensions, not mounted on a sound intensity probe. Figure 8 shows such a comparison for axial incidence, for incidence perpendicular to the probe, and for random (diffuse) inci-

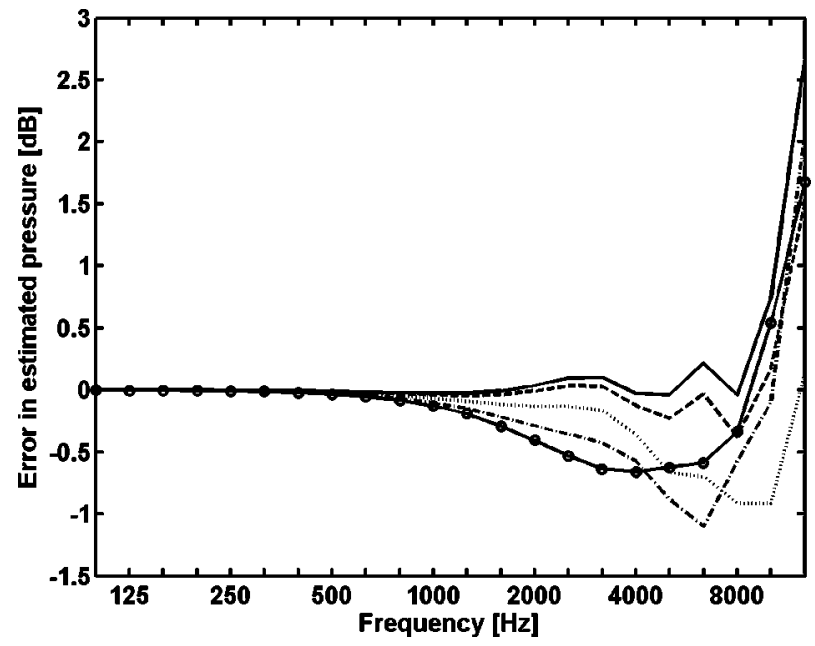

FIG. 7. Error in the estimated sound pressure with $w=0.775$ for a plane wave at various angles of incidence:-, $0^{\circ} ;---, 20^{\circ} ; \cdots, 40^{\circ} ;-\cdots \cdot-\cdot 60^{\circ}$; $-\mathrm{O}_{-}, 80^{\circ}$.

dence. The diffuse-field response has been calculated as a weighted average of the squared response at all angles of incidence. It is apparent that the intensity probe with the improved sound pressure estimate is much more omnidirectional than an ordinary microphone.

Sound intensity probes are sometimes used relatively near sources of sound. Figures 9(a), 9(b), and 9(c) show the error in the estimated sound pressure generated by a point source placed on the axis of the probe at distances between 10 and $100 \mathrm{~cm}$ from its center, calculated with $w=0.225$, $w=0.775$, and $w=0.5$. It is apparent that $w=0.225$ leads to underestimation and $w=0.775$ leads to overestimation if the point source is near the probe, in agreement with the fact that a weighting factor of 1 simply shifts the measurement position $6 \mathrm{~mm}$ closer to the source whereas a factor of 0 does the opposite. In this respect the conventional estimate with $w$ $=0.5$ is clearly better, although even this one is not perfect. In all cases the errors are essentially independent of the frequency.

The measured pressure-intensity index is normally used

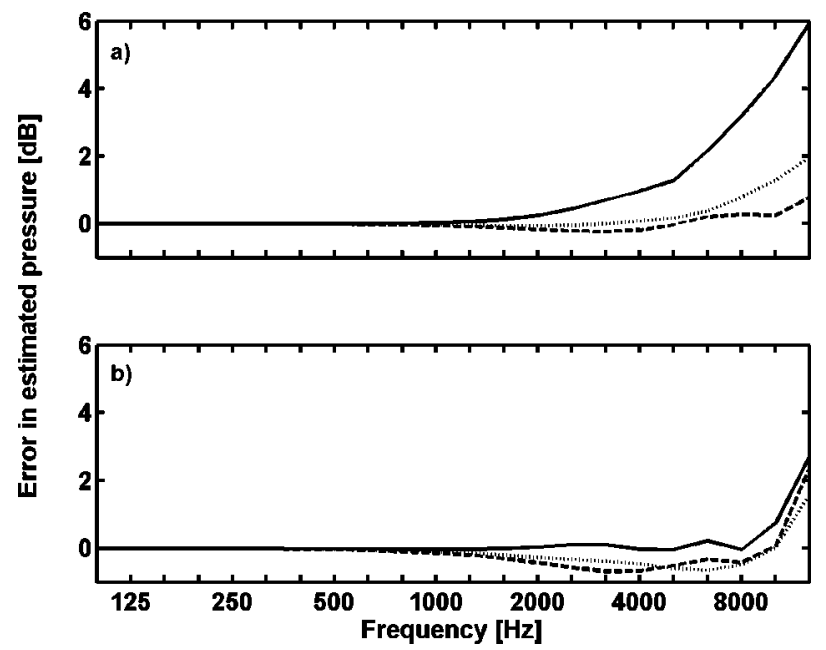

FIG. 8. Error in the estimated sound pressure calculated for (a) a solitary 0.5 in. microphone, and (b) the optimized sound intensity probe, with $w$ $=0.775 .-, 0^{\circ} ;---, 90^{\circ} ; \cdots$, diffuse incidence. 


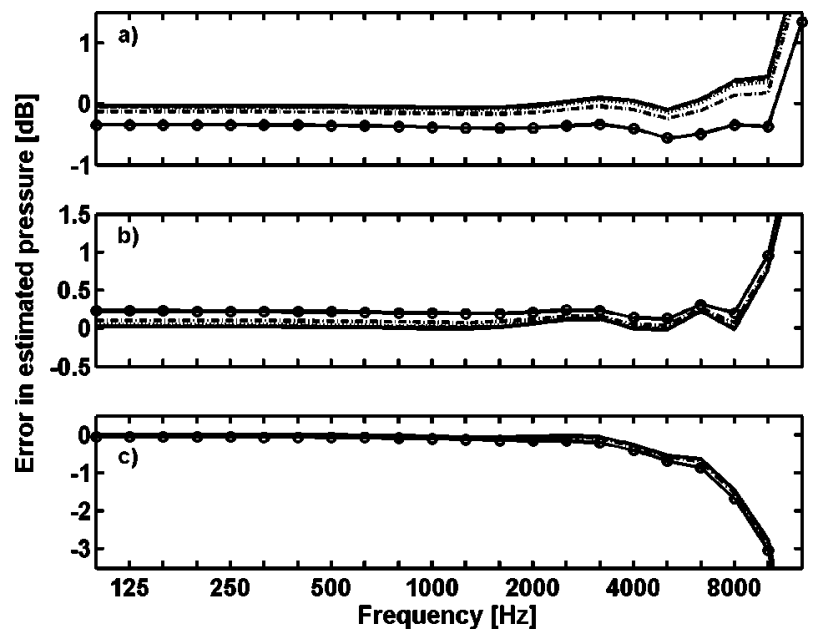

FIG. 9. Error in the estimated sound pressure generated by a point source, estimated with (a) $w=0.225$, (b) $w=0.775$, and (c) $w=0.5$. Distance between the point source and the center of the intensity probe:-, $1 \mathrm{~m} ;---, 0.75$ $\mathrm{m} ; \cdots, 0.5 \mathrm{~m} ; \cdots,-\cdot, 0.25 \mathrm{~m} ;-\bigcirc_{-,} 0.1 \mathrm{~m}$.

for giving an indication of phase mismatch errors in the sound intensity, owing to the fact that phase mismatch gives rise to an error in the measured intensity that is proportional to the mean square pressure. ${ }^{1}$ In the circumstances the pressure estimate with $w=0.775$, which overestimates the pressure slightly when the source is near and thus gives a conservative estimate of the phase mismatch error, is a better choice than $w=0.225$. Nearby sources behind the intensity probe are unlikely to occur in normal use.

Microphones for measurement of sound intensity must be phase matched fairly well, and state-of-the-art sound intensity microphones are matched to a maximum phase response difference of $0.05^{\circ}$ below $250 \mathrm{~Hz}$ and a phase difference proportional to the frequency above $250 \mathrm{~Hz}$ (say, $0.2^{\circ}$ at $1 \mathrm{kHz}) .{ }^{13-15}$ This is a consequence of the fact that phase mismatch in most of the frequency range is caused by differences between the resonance frequencies and the damping of the two microphones. ${ }^{16}$

The influence of the phase mismatch on measurement of sound pressure with an intensity probe has been examined by artificially changing the phase of one of the pressure signals. The influence of the phase error of a matched microphone set is completely negligible. Figures 10 (a) and 10(b), which correspond to Figs. 5 and 7, show the influence of the typical phase mismatch of unmatched microphones $\left(25^{\circ}\right.$ at $\left.10 \mathrm{kHz}\right)$ for $w=0.5$ and $w=0.775$. As can be seen the influence of such phase mismatch is fairly moderate, and the improved estimate is less sensitive than the conventional one. This is in good agreement with the fact that the effect of the phase error must disappear altogether when $w=0$.

\section{Discussion}

The main purpose of this work has been to examine whether it is possible to measure the sound pressure and thus the pressure-intensity index reliably in the frequency range where the sound intensity itself can be measured reliably, that is, up to $10 \mathrm{kHz}$ for an intensity probe with two $0.5 \mathrm{in}$.

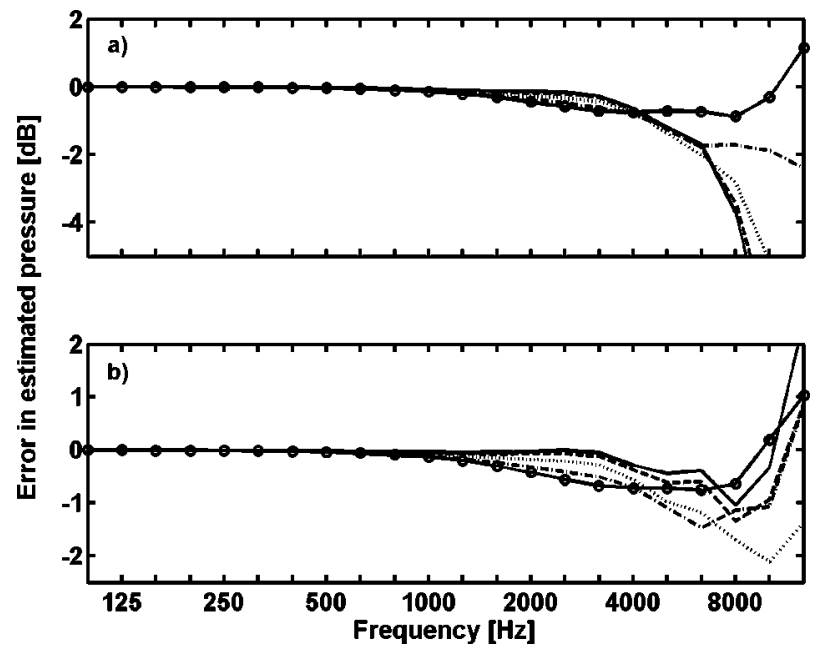

FIG. 10. Error in (a) the conventional estimate of the sound pressure ( $w$ $=0.5)$ and $(\mathrm{b})$ in the improved estimate $(w=0.775)$ for a plane wave at various angles of incidence when phase mismatch corresponding to unmatched microphones has been introduced:-, $0^{\circ} ;---, 20^{\circ} ; \cdots, 40^{\circ} ;-\cdots-$, $60^{\circ} ;-\bigcirc-, 80^{\circ}$.

microphones separated by a $12 \mathrm{~mm}$ spacer. It seems to be possible to improve the conventional estimate considerably in a very simple manner.

The largest error of the improved estimate occurs at 6.3 $\mathrm{kHz}$ at an angle of incidence of $60^{\circ}$. Under such conditions the sound pressure will be underestimated by about $1 \mathrm{~dB}$. However, the conventional method underestimates the sound pressure by $1.2 \mathrm{~dB}$ under the same conditions and is much worse than the improved one for axial incidence.

It is not possible to improve the performance for perpendicular incidence, simply because the two microphone signals are identical in phase as well as in amplitude. By contrast, it is possible to improve the estimate in a diffuse sound field. Although the two pressure signals have the same magnitude in a diffuse sound field and the resulting phase difference is zero the signals are not completely coherent. ${ }^{17}$ In such a sound field $w=0.14$ gives slightly better results than $w=0.775$. However, the improvement is modest indeed (less than $0.2 \mathrm{~dB}$ ), and the performance for axial incidence is worsened significantly: the maximum error is almost doubled. A weighting with $w=0.775$ seems to be the best overall solution.

The spacer length assumed in the calculations has been optimized for measurement of sound intensity, not sound pressure. In some respects it is more difficult to measure the sound pressure at high frequencies: as demonstrated by Fig. 1 the finite-difference error is larger, and whereas large relative errors in the estimated intensity are not very important near perpendicular incidence all directions are equally important in sound pressure measurements. Increasing the length of the spacer would not have any influence on the estimated pressure when the probe is exposed to perpendicular sound incidence, but it would reduce the estimated pressure at high frequencies for sound incidence near the axis of the probe. Such considerations and the results presented in Figs. 7 and 8 lead to the conclusion that it is unlikely that the performance could be improved appreciably by choosing an- 
other spacer length—quite apart from the fact that this would be unacceptable if the probe is to be used for measurement of sound intensity.

The results have indicated that a two-microphone arrangement could be advantageous even in ordinary sound pressure measurements. It may seem paradoxical to suggest measuring sound pressure with an intensity probe. The conventional solution in ordinary sound pressure measurements is to use microphones designed to have a flat or nearly flat frequency response under conditions that are similar to those of the measurement. For example, "free-field microphones" are designed to have a flat response for axial incidence (at the expense of the response under other conditions), ${ }^{18}$ and "random incidence microphones" are designed to have a flat response in a diffuse sound field. ${ }^{18}$ However, the comparison with an ordinary microphone (one not mounted on an intensity probe) has demonstrated that a two-microphone arrangement with the proposed weighting is far better in the general case. Thus such a two-microphone arrangement may have a potential in conventional sound pressure measurements not related with sound intensity. A two-microphone sound level meter would be far simpler than a sound intensity analyzer and would not require phase-matched microphones.

\section{EXPERIMENTAL RESULTS}

To examine the validity of the numerical results presented in the foregoing a few experiments have been carried out. A frequency analyzer of type Brüel \& Kjær 3550 was used in combination with a sound intensity probe of type B\&K 3548, either with 0.5 in. microphones of type B\&K 4181 and a $12 \mathrm{~mm}$ spacer or with a $0.25 \mathrm{in}$. microphone of type $\mathrm{B} \& \mathrm{~K} 4178$ on one of the 0.25 in. preamplifiers taken out of the probe. Both microphone sets are free-field microphones. Therefore, it is necessary to compensate for the drop in the pressure sensitivities at high frequencies when the pressure response is needed. This was done using frequency responses measured with an electrostatic actuator, as described in Ref. 3. The weighted estimate of the sound pressure level was calculated from the two power spectra $S_{11}$ and $S_{22}$ and the real part of the cross spectrum $C_{12}$ as follows:

$$
L_{p}\left(\omega_{0}\right)=10 \log \left(\overline{p_{w}^{2}}\left(\omega_{0}\right)\right),
$$

where

$$
\begin{aligned}
\overline{p_{w}^{2}}\left(\omega_{0}\right)= & \frac{w^{2}}{\pi} \int_{\omega_{0}-\Delta \omega / 2}^{\omega_{0}+\Delta \omega / 2} S_{11}(\omega) d \omega \\
& +\frac{(1-w)^{2}}{\pi} \int_{\omega_{0}-\Delta \omega / 2}^{\omega_{0}+\Delta \omega / 2} S_{22}(\omega) d \omega \\
& +\frac{2 w(1-w)}{\pi} \int_{\omega_{0}-\Delta \omega / 2}^{\omega_{0}+\Delta \omega / 2} C_{12}(\omega) d \omega .
\end{aligned}
$$

Here the power spectra and the cross power spectrum are two-sided spectra, $\omega_{0}$ is the center frequency, and $\Delta \omega$ is the bandwidth.

The experimental results shown in Fig. 11 have been determined in an anechoic room about $2 \mathrm{~m}$ from a loudspeaker driven with white noise. The "true" sound pressure



FIG. 11. Error in the estimated sound pressure for (a) axial incidence and (b) perpendicular incidence. - , conventional estimate $(w=0.5)$; $\bigcirc$, corresponding measurement; ---, improved estimate $(w=0.775) ; \times$, corresponding measurement; $\cdots$, front microphone $(w=1)$.

was measured using the 0.25 in. microphone of type $\mathrm{B} \& \mathrm{~K}$ 4178 , not corrected for its pressure response, pointing towards the loudspeaker. Figure 11(a) compares calculated and measured pressure responses for axial incidence using the conventional weighting factor $w=0.5$ and the proposed one, $w=0.775$. There is agreement within $\pm 1 \mathrm{~dB}$, and the superiority of the improved estimate is apparent. The calculated response of the front microphone is also shown.

Figure 11(b) shows a similar comparison for perpendicular incidence. In this case the three calculated curves coincide, and the experimental results are also almost unaffected by the weighting factor, which indicates that the physical sound intensity probe is almost symmetrical. The agreement between measurements and calculations is within $\pm 1.2 \mathrm{~dB}$.

The experimental results shown in Fig. 12 have been obtained in a large reverberation room about $5 \mathrm{~m}$ from the



FIG. 12. Error in the estimated sound pressure for diffuse incidence.conventional estimate $(w=0.5)$; $\bigcirc$, corresponding measurement; ---, improved estimate $(w=0.775) ; \times$, corresponding measurement; $\cdots$, front microphone $(w=1)$. 
loudspeaker. In this case the "true" sound pressure was measured with the $0.25 \mathrm{in}$. microphone of type B\&K 4178, this time corrected for its pressure response. This should be accurate up to $10 \mathrm{kHz}$, as can be seen by scaling the diffusefield response of 0.5 in. microphones shown in Fig. 8. Figure 12 compares calculated and measured pressure responses using the conventional weighting factor $w=0.5$ and the proposed one, $w=0.775$. There is agreement within $\pm 0.5 \mathrm{~dB}$. The calculated response of the front microphone is also shown.

\section{CONCLUSIONS}

A numerical and experimental study of measurement of sound pressure with two-microphone sound intensity probes has demonstrated that the conventional estimate based on the arithmetic mean of the two pressure signals can be improved significantly by applying different weighting factors on the two signals. The optimum length of the spacer between the microphones for sound intensity measurements is about one microphone diameter. The corresponding optimum values of the weighting factors for sound pressure measurements are about 0.2 and 0.8 , respectively, and the latter is slightly better when the source is near the probe. With 0.5 in. microphones and a $12 \mathrm{~mm}$ spacer the improved estimate gives tolerable accuracy under a variety of sound field conditions up to 10 $\mathrm{kHz}$, which is about an octave higher than the conventional estimate.

The proposed improvement makes an intensity probe with 0.5 in. microphones more omnidirectional than a single 0.25 in. microphone and may therefore be useful also in sound pressure measurements not related to sound intensity.

${ }^{1}$ F. J. Fahy, Sound Intensity, 2nd ed. (E \& FN Spon, London, 1995), See sections 5.3, 6.2, 7.2, and 9.3.

${ }^{2}$ G. Rasmussen and M. Brock, "Acoustic intensity measurement probe," in Proceedings of Recent Developments in Acoustic Intensity Measurement, edited by M. Bockhoff (Senlis, France, 1981), pp. 81-88.

${ }^{3}$ F. Jacobsen, V. Cutanda, and P. M. Juhl, "A numerical and experimental investigation of the performance of sound intensity probes at high frequencies," J. Acoust. Soc. Am. 103, 953-961 (1998).

${ }^{4} \mathrm{~F}$. Jacobsen, "A simple and effective correction for phase mismatch in intensity probes," Appl. Acoust. 33, 165-180 (1991).

${ }^{5}$ S. E. Keith, G. Krishnappa, V. Chiu, "The potential for extending the frequency range of sound intensity standards," in Proceedings of the Fifth European Conference on Noise Control, edited by G. Brambilla, C. Ianiello, and L. Maffei (Naples, Italy, 2003), Paper 169-IP.

${ }^{6}$ T. J. Schultz, "Acoustic wattmeter," J. Acoust. Soc. Am. 28, 693-699 (1956).

${ }^{7}$ P. Juhl, "A numerical investigation of standard condenser microphones," J. Sound Vib. 177, 433-446 (1994).

${ }^{8}$ V. Cutanada, P. M. Juhl, and F. Jacobsen, "On the modeling of narrow gaps using the standard boundary element method," J. Acoust. Soc. Am. 109, 1296-1303 (2001).

${ }^{9}$ V. Cutanda, P. M. Juhl, and F. Jacobsen, "A numerical investigation of the performance of sound intensity probes at high frequencies," in Proceedings of Fourth International Congress on Sound and Vibration, edited by M. J. Crocker (St. Petersburg, Russia, 1996), pp. 1897-1904.

${ }^{10}$ H. A. Schenck, "Improved integral formulation for acoustic radiation problems," J. Acoust. Soc. Am. 44, 41-58 (1968).

${ }^{11} \mathrm{P}$. Juhl, "A numerical study of the coefficient matrix of the boundary element method near characteristic frequencies," J. Sound Vib. 175, 39-50 (1994).

${ }^{12}$ E. Frederiksen and M. Piil, "Characteristics of microphone pairs and probes for sound intensity measurements," Brüel \& Kjærum, Report BA077-11, 1987.

${ }^{13}$ International Electrotechnical Commission (IEC) Standard 61043, "Instruments for the measurement of sound intensity-Measurements with pairs of pressure sensing microphones," 1993.

${ }^{14}$ American National Standard ANSI S1.9-1996, "Instruments for the measurement of sound intensity," 1996.

15 "Product data, sound intensity pair-type 4197," Brüel \& Kjær, Nærum, Denmark, 2000.

${ }^{16}$ E. Frederiksen and O. Schultz, "Pressure microphones for intensity measurements with significantly improved phase properties," Brüel \& Kjær Technical Review 4, 11-19 (1986).

${ }^{17}$ F. Jacobsen and T. Roisin, "The coherence of reverberant sound fields," J. Acoust. Soc. Am. 108, 204-210 (2000).

${ }^{18}$ R. W. Krug, "Sound level meters," in Handbook of Acoustics, edited by M. J. Crocker (Wiley, New York, 1998), Chap. 105. 